\title{
SIMULTANEOUS BILATERAL AVULSION FRACTURE OF THE TIBIAL TUBEROSITY IN A TEENAGER: CASE REPORT AND THERAPY USED
}

Rodrigo Pires e Albuquerque', Vincenzo Giordano ${ }^{2}$, Antônio Carlos Pires Carvalho ${ }^{3}$, Thiago Puell, Maria Isabel Pires e Albuquerque ${ }^{5}$, Ney Pecegueiro do Amaral ${ }^{6}$

\section{ABSTRACT}

Simultaneous bilateral avulsion fracture of the tibial tuberosity in teenagers is a rare lesion. We describe the first case in the literature, in a teenage girl who sustained a fall while jumping during a volleyball match. No predisposing factors were iden- tified. The lesions were treated with open surgical reduction and internal fixation. The aim of the present study was to present a case of simultaneous bilateral avulsion fracture of the tibial tuberosity in a teenage girl and the therapy used.

Keywords - Fractures, Bone; Tibia; Volleyball; Adolescence

\section{INTRODUCTION}

Avulsion fractures of the tibial tuberosity are rare injuries accounting for $1 \%$ of all growth plate injuries $^{(1)}$. Bilateral occurrence of fractures of the tibial tuberosity, suffered simultaneously, makes these injuries even rarer.

This type of injury occurs in individuals with an immature skeleton, in which the muscles, ligaments and tendons are generally stronger than the growth plates. For this reason, it is rare to see a tear in tendon material in children or adolescents.

The aim of this study was to present the first case of simultaneous bilateral avulsion fracture of the tibial tuberosity in a female, and the therapy used.

\section{CASE REPORT}

A healthy 13-year-old adolescent girl who fell while jumping during a volleyball match evolved immediately with pain, hemarthrosis, hematoma and inability to walk (Figure 1). The patient was sent to the emergency service of our hospital. Physical examination revealed bilateral edema in the knees and a high patella. She also presented inability to actively extend her leg. At the time of the trauma, the patient weighed $84 \mathrm{~kg}$ and her height was $1.65 \mathrm{~m}$.

Radiographs on the knees showed bilateral avulsion fractures of the tibial tubercle, thus confirming the diagnosis (Figure 2). The patient's clinical history was investigated and blood tests were performed for all the markers for rheumatic and renal diseases. From these, relationships with systemic disease or steroid use were rejected. Osgood-Schlatter disease was also ruled out. In the right knee, the fracture was classified as type III, and in the left knee, as type II, using the Watson-Jones descriptions ${ }^{(2)}$.

The surgical procedures were performed one day after hospitalization, using tourniquets and straight anterior incisions in the knees. The surgical technique used consisted of open reduction and osteosynthesis using $6.5 \mathrm{~mm}$ cannulated screws (thread 16), without the need for reinforcement in either knee, since

1 - Coordinator of the Knee Surgery Sector of the Miguel Couto Municipal Hospital, Rio de Janeiro, RJ, Brazil.

2 - Coordinator of the Medical Residence Program of the Miguel Couto Municipal Hospital, Rio de Janeiro, RJ, Brazil.

3 - Associate Professor in the Department of Radiology, Federal University of Rio de Janeiro, RJ, Brazil.

4 - Collaborating Orthopedist in the Orthopedics and Traumatology Service, Miguel Couto Municipal Hospital, Rio de Janeiro, RJ, Brazil.

5 - Pediatrician in the National Cancer Institute, Rio de Janeiro, RJ, Brazil.

6 - Head of the Orthopedics and Traumatology Service, Miguel Couto Municipal Hospital, Rio de Janeiro, RJ, Brazil

Work performed at the Professor Nova Monteiro Orthopedics and Traumatology Service, Miguel Couto Municipal Hospital (SOT-HMMC), Rio de Janeiro, RJ, Brazil. Correspondence: Av. Henrique Dodsworth 83, ap. 105, Copacabana, 22061-030, Rio de Janeiro, RJ, Brazil. E-mail: rodalbuquerque@ibest.com.br Work received for publication: May 13, 2011; accepted for publication: June 15, 2011. 


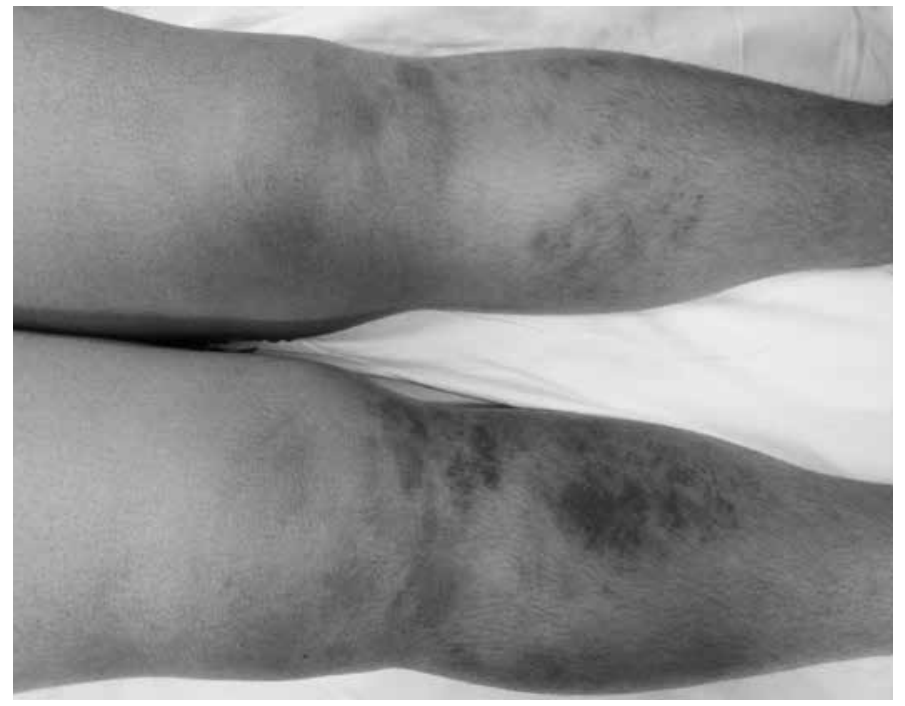

Figure 1 - Bilateral hematoma of the knees.

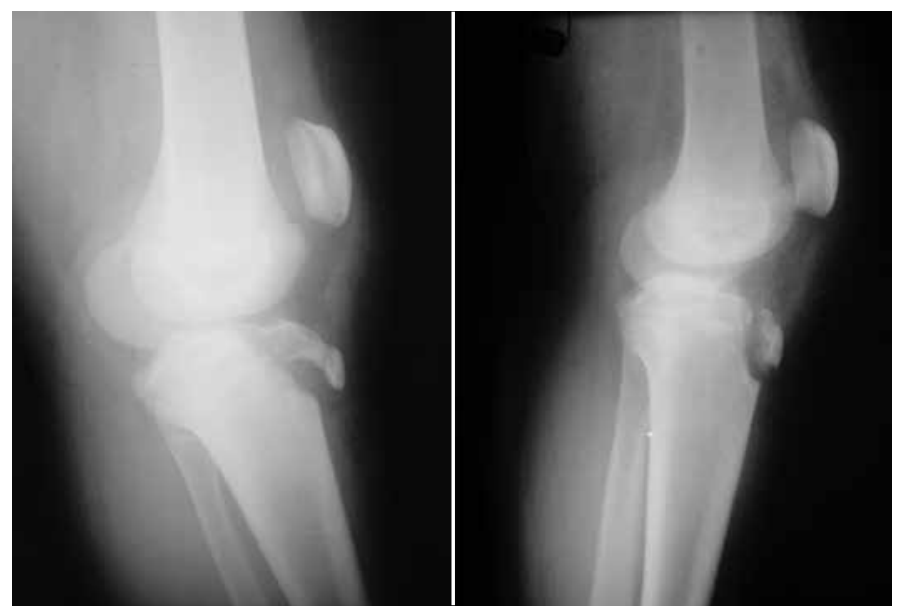

Figure 2 - Radiographs on both knees, before the operation.

these were acute traumatic lesions (Figure 3). The osteosynthesis was tested by means of careful flexion of the knee joint.

After the operation, both knees were immobilized using a long knee immobilizer for six weeks, which was removed for active rehabilitation exercises in order to avoid atrophy of the quadriceps. The program consisted of isometric exercises for the quadriceps during the immobilization period and active exercises for the quadriceps with progressive increase of the range of motion. The complete range of motion and full functioning of the knees were attained after four months.

Our patient was evaluated at one week, 15 days, one month, 45 days, two months and then monthly until six months after the operation, with three-monthly consultations thereafter. Our follow-up with this patient has been for two years, and she has returned to her habitual activities. The follow-up has included radiographic control examinations, to assess the fracture consolidation and any growth disorders. For the functional evaluations on the knees, we used the modified Lysholm system ${ }^{(3)}$. We achieved mean scores of 95 points for the left knee and 96 points for the right knee, which are considered to be excellent averages according to this assessment system (Figure 4).

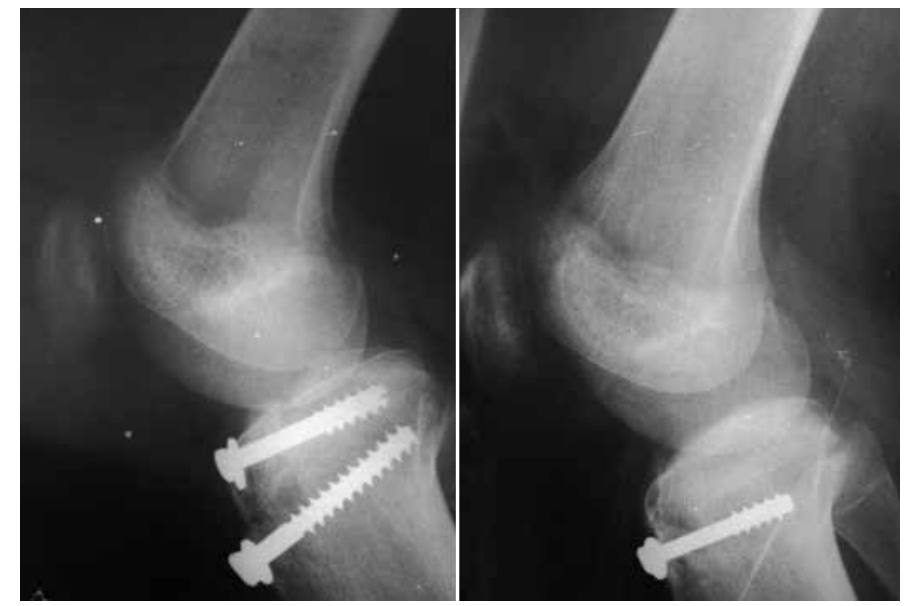

Figure 3 - Radiographs on both knees, after the operation.

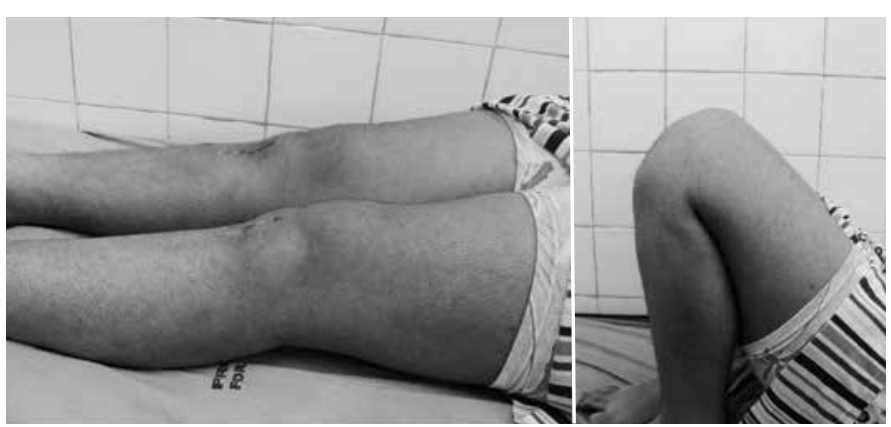

Figure 4 - Postoperative functional assessment.

\section{DISCUSSION}

In relation to sex, the literature shows that males predominate $^{(4)}$. This case report is the first in which a bilateral avulsion fracture of the tibial tubercle in a female was described, thus demonstrating the rarity of such injuries.

The literature shows a relationship with sports practice, and we corroborate and affirm this thinking ${ }^{(4)}$. Our patient was an amateur volleyball player. Furthermore, she was 13 years of age, which was in line with the mean age reported in the literature ${ }^{(5,6)}$.

The usual mechanism for avulsion fractures of the tibial tubercle is a sudden contraction of the quadriceps during knee extension or rapid passive knee flexion against a contraction of the quadriceps ${ }^{(1)}$. 
There are studies that have correlated avulsion fractures of the tibial tubercle with Osgood-Schlatter disease as a predisposing factor ${ }^{(5)}$. Our patient said that she had not had any previous problems in either knee. One report in the literature correlated avulsion fractures of the tibial tubercle with associated lesions in the $\mathrm{knees}^{(6)}$. Careful examination on our patient did not demonstrate any associated lesion.

With regard to complementary examinations, radiography on the knee provided good accuracy of diagnostic confirmation, as well as being a low-cost procedure. Computed tomography is well indicated when there is a fracture of the tibial tubercle that affects the joint surface ${ }^{(7,8)}$.

There is some controversy regarding whether to keep the patient immobilized after the operation, for how long to do this ${ }^{(9)}$. It needs to be borne in mind that this type of injury generally affects individuals with an immature skeleton. Among this population, there is a greater risk that medical orders will be disregarded ${ }^{(10)}$. On the other hand, individuals with an immature skeleton present a lower risk of joint stiffness, compared with the adult population. For this reason, we advocate that a brace should be used for six weeks, which should be taken off every day for exercises aimed at gaining range of motion and developing the musculature.

The main aims of the treatment are to diagnose the lesion, achieve early repair and preserve the growth plates, in order to achieve a better functional result.

Occurrences of simultaneous bilateral avulsion fracture of the tibial tuberosity in females are rare, and the present case is the first described in the worldwide literature. Our patient was followed up for two years after the operation, with an excellent result according to the modified Lysholm scoring system.

\section{REFERENCES}

1. Hamilton SW, Gibson PH. Simultaneous bilateral avulsion fractures of the tibial tuberosity in adolescence: A case report and review of over 50 years of literature. Knee. 2006;13(5):404-7.

2. Watson-Jones R. Knee fractures. In: Watson-Jones. Fractures and joint injuries 5th ed. Baltimore: Williams \& Wilkins; 1976.

3. Tegner Y, Lysholm J. Rating systems in the evaluation of knee ligament injuries. Clin Orthop Relat Res. 1985;(198):43-9.

4. Hanley C, Roche SJ, Chhabra J. Acute simultaneous bilateral avulsion fractures of the tibial tubercles in a 15-year-old male hurler: case report and literature review. Ir J Med Sci. 2011;180(2):589-92.

5. Arredondo-Gómez E, López Hernández JD, Chávez Martínez F. [Fracture due to bilateral avulsion of the tuberosity of the shin bone (tibia). A case report]. Acta Ortop Mex. 2007;21(3):154-8.
6. McKoy BE, Stanitski CL, Hartsock LA. Bilateral tibial tubercle avulsion fractures with unilateral recurrence. Orthopedics. 2006;29(8):731-3.

7. Tulic G, Sopta J, Bumbasirevic M, Todorovic A, Vucetic C. Simultaneous bilateral avulsion fracture of the tibial tubercle in adolescent: a case report. J Pediatr Orthop B. 2010;19(1):118-21.

8. Slobogean GP, Mulpuri K, Alvarez CM, Reilly CW. Comminuted simultaneous bilateral tibial tubercle avulsion fractures: a case report. J Orthop Surg (Hong Kong). 2006;14(3):319-21.

9. Ergün M, Taşkiran E, Ozgürbüz C. Simultaneous bilateral tibial tubercle avulsion fracture in a basketball player. Knee Surg Sports Traumatol Arthrosc. 2003;11(3):163-6.

10. Lepse PS, McCarthy RE, McCullough FL. Simultaneous bilateral avulsion fracture of the tibial tuberosity. A case report. Clin Orthop Relat Res. 1988;(229):232-5. 